\title{
Erratum to: Communications and Multimedia Security Issues of the New Century
}

\author{
Ralf Steinmetz, Jana Dittman, and Martin Steinebach \\ German National Research Center for Information Technology, \\ Institute IPSI, Germany
}

\section{Erratum to:}

R. Steinmetz et al. (Eds.)

Communications and Multimedia Security Issues of the New Century

DOI: $10.1007 / 978-0-387-35413-2$

The book was inadvertently published with an incorrect name of the copyright holder. The name of the copyright holder for this book is: (c) IFIP International Federation for Information Processing. The book has been updated with the changes. 